\title{
VLBI multi-epoch water maser observations toward massive protostars
}

\author{
José M. Torrelles ${ }^{1}$, José F. Gómez ${ }^{2}$, Nimesh A. Patel ${ }^{3}$, \\ Salvador Curiel ${ }^{4}$, Guillem Anglada ${ }^{2}$, and Robert Estalella ${ }^{5}$ \\ ${ }^{1} \mathrm{ICE}(\mathrm{CSIC})-\mathrm{UB} / \mathrm{IEEC}$, Barcelona (Spain) \\ ${ }^{2}$ IAA(CSIC), Granada (Spain) \\ ${ }^{3}$ Harvard-Smithsonian, CfA, Cambridge (USA) \\ ${ }^{4}$ IAUNAM, México D.F. (México) \\ ${ }^{5}$ UB/IEEC, Barcelona (Spain)
}

\begin{abstract}
VLBI multi-epoch water maser observations are a powerful tool to study the gas very close to the central engine responsible for the phenomena associated with the early evolution of massive protostars. In this paper we present a summary of the main observational results obtained toward the massive star-forming regions of Cepheus A and W75N. These observations revealed unexpected phenomena in the earliest stages of evolution of massive objects (e.g., non-collimated "short-lived" pulsed ejections in different massive protostars), and provided new insights in the study of the dynamic scenario of the formation of high-mass stars (e.g., simultaneous presence of a jet and wide-angle outflow in the massive object Cep A HW2, similar to what is observed in low-mass protostars). In addition, with these observations it has been possible to identify new, previously unseen centers of high-mass star formation through outflow activity.
\end{abstract}

Keywords. ISM: general, stars: formation, ISM: jets and outflows

\section{Introduction}

It is well-established that low-mass stars form via an accretion process. A natural consequence of this process is the formation, during the early stages of evolution of a young stellar object (YSO), of a system (with typical scales of $\simeq 100 \mathrm{AU}$ ) that comprises a central protostar, surrounded by a circumstellar (protoplanetary) disk, and a collimated outflow, ejected perpendicular to the disk (e.g., Anglada 1996). The disk is the reservoir of material from which the central protostar accretes further matter, while the collimated outflow releases the necessary angular momentum and magnetic flux for this accretion to proceed. This accretion scenario seems to be generally applicable in the formation of stars up to $\simeq 20 \mathrm{M}_{\odot}$ (e.g., Garay \& Lizano 1999). However, very few massive protostar-diskoutflow systems have been identified and studied in detail, at scales $\leqslant 3000$ AU (Patel et al. 2005, Jiménez-Serra et al. 2007, Torrelles et al. 2007, Zapata et al. 2009, Davies et al. 2010, Carrasco-González et al. 2010a, 2011, Fernández-López et al. 2011). This scarcity of studies is probably due to observational limitations (sensitivity and angular resolution), given that high-mass stars are more rare, are typically located at larger distances, and form in a more clustered environment than their low-mass counterparts.

Observations of water maser emission $(\lambda=1.35 \mathrm{~cm})$ towards massive YSOs can overcome these observational limitations (sensitivity and angular resolution). Water maser emission is compact ( $\leqslant 1$ mas) and strong (brightness temperatures can reach $10^{10} \mathrm{~K}$ ), which make it an ideal tool for observations with Very Long Baseline Interferometry (VLBI), i.e., with angular resolutions $<1$ mas. Therefore, we can study shocked, warm 
$(\sim 500 \mathrm{~K})$, and dense $\left(\simeq 10^{8}-10^{9} \mathrm{~cm}^{-3}\right)$ gas, at scales of only 10-1000 AU from massive protostars (Reid \& Moran 1981), with the possibility of accurately tracing their kinematics, by measuring proper motions of a few $\mathrm{km} \mathrm{s}^{-1}$ in timescales of a few weeks. In this paper we summarize the main results of our studies obtained toward the high-mass star forming regions of Cepheus A and W75N, using VLBI multi-epoch water maser observations with an angular resolution of $\sim 0.5$ mas. These results were reported by Torrelles et al. (2001a,b, 2003, 2011) and summarized in Torrelles et al. (2012). Other very interesting examples (not included in this paper) can be found in the studies on IRAS 06061+2151 (Motogi et al. 2008), AFGL 5142 (Goddi, Moscadelli, \& Sanna 2011), AFGL 2591 (Sanna et al. 2012, Trinidad et al. 2003), and Cep A HW3d (Chibueze et al. 2012) (see also talks in this conference by A. Bartkiewicz-H. van Langevelde, J. Chibueze, C. Goddi, T. Hirota, and A. Sanna).

\section{Cepheus A}

Cepheus $\mathrm{A}$ is an active high-mass star-forming region, located at only $700 \mathrm{pc}$ (Moscadelli et al. 2009), which contains a cluster of at least 16 sources within $\simeq 30^{\prime \prime}$ (Hughes \& Wouterloot 1984, Garay et al. 1996). Its star-formation activity is dominated by the source HW2. The radio continuum emission in HW2 seems to trace an ionized jet, powered by a massive protostar $\left(\simeq 15-20 \mathrm{M}_{\odot}\right)$. Extremely broad millimeter wavelength hydrogen recombination lines were recently detected toward this source with terminal velocity $>500 \mathrm{~km} \mathrm{~s}^{-1}$ for the ionized gas (Jiménez-Serra et al. 2011). This source was the first identified case of a disk-protostar-jet system in a massive YSO, at scales of $\simeq 1000$ AU (Rodríguez et al. 1994, Patel et al. 2005, Curiel et al. 2006, Jiménez-Serra et al. 2007, Torrelles et al. 2007, Vlemmings et al. 2010). This suggests that this high-mass object formed by an accretion mechanism, in a similar way to low-mass stars.

HW2 is associated with strong water maser emission, which has been studied in detail with the Very Large Array (VLA, beam size $\simeq 80$ mas) and the Very Long Baseline Array (VLBA, beam size $\simeq 0.5$ mas), for a total of 9 different epochs (Torrelles et al. 2001a,b, 2011). Fig. 1 shows the location of the water masers, overlaid on the $1.3 \mathrm{~cm}$ continuum map of the HW2 radio jet and the nearby HW3c and HW3d objects (located $\sim 3^{\prime \prime}$ south from HW2; see Chibueze et al. 2012 and Chibueze's talk in this conference for a detailed study of the distribution and kinematics of the water masers associated with HW3d). The VLBA data showed that individual maser features tend to be organized both spatially and kinematically, forming linear microstructures of a few mas in size. The flattened appearance of these water maser linear "microstructures" and their proper motions indicate that they are originated through shock excitation by outflows, as expected from theory (e.g., Elitzur et al. 1992). These linear microstructures are the building blocks of larger, coherent linear/arcuate structures of $\simeq 40-100$ mas. The identified structures in the neighborhood of HW2 are in the regions labelled R1 to R8 in Figure 1.

In particular, the masers in the $\mathrm{R} 4$ subregion trace a section of a nearly elliptical ring of $\simeq 70$ mas size (50 AU), with expanding motions of $\sim 15-30 \mathrm{~km} \mathrm{~s}^{-1}$ (Figure 2), from which an extremely short dynamical age of 4-8 year can be derived. This expansion must be driven by a yet undetected probably massive YSO (to explain the high luminosity of the water masers), located at the geometrical center of the ring, $\sim 130$ AU from HW2. A detailed inspection of Fig. 2 also shows some internal structure within the ring in individual epochs, with several "shells" that may have been created by multiple successive ejections. Future sensitive $\mathrm{cm}$ and (sub)mm observations may help us to identify the massive object powering this structure. 


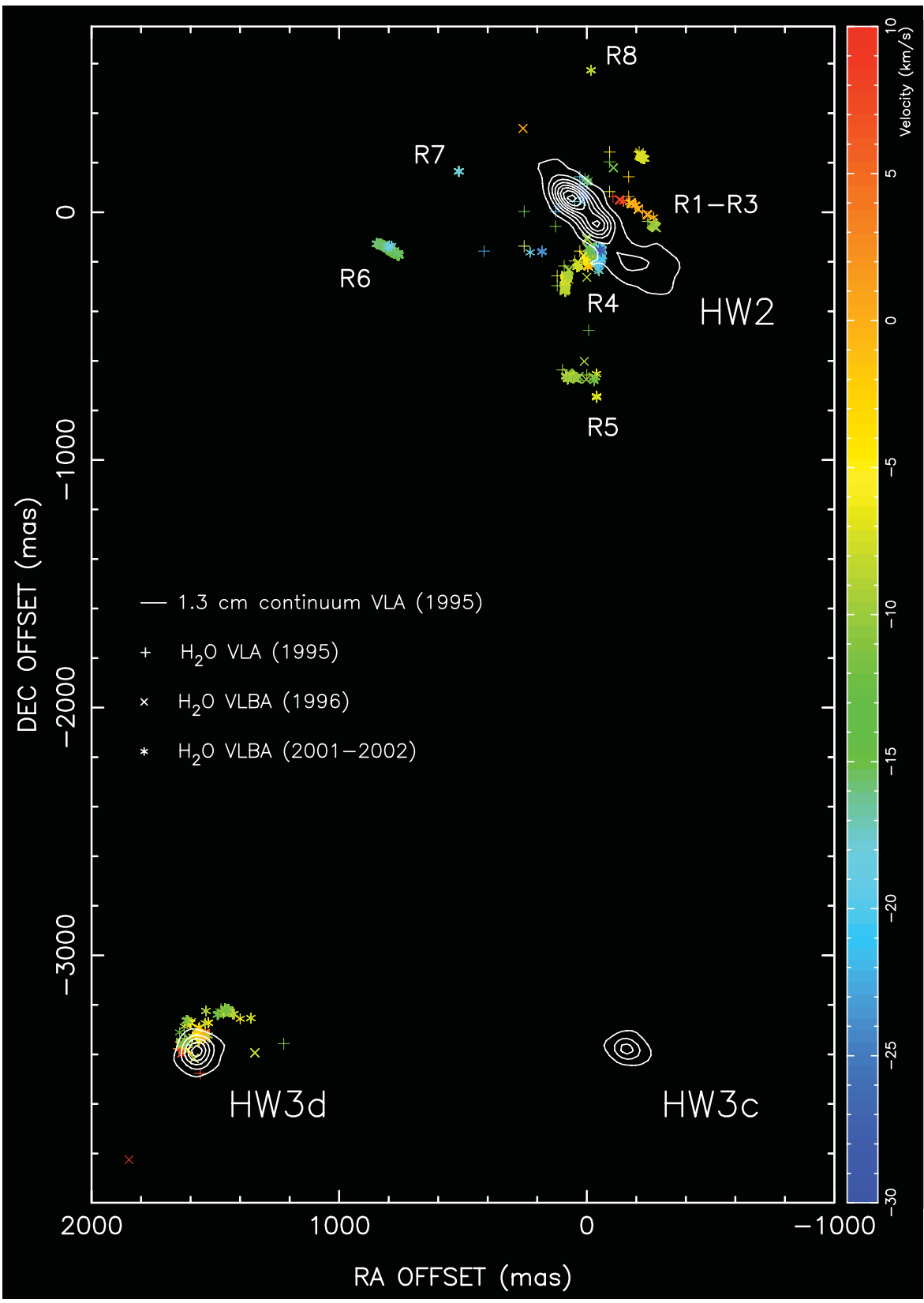

Figure 1. (Published online) Positions and radial velocities (color code) of the $\mathrm{H}_{2} \mathrm{O}$ masers overlaid onto the $1.3 \mathrm{~cm}$ continuum maps (contours) of Cep A HW2, HW3c, and HW3d. Sub-regions "R" discussed in this paper are numbered. (Figure from Torrelles et al. 2011).

Another remarkable subregion is R5, located $\sim 6^{\prime \prime}$ south of HW2. In our 1996 observations, the masers in this region traced part of a nearly perfect circle (to an accuracy of $1 / 1000$ ), and expanding at $9 \mathrm{~km} \mathrm{~s}^{-1}$. We interpreted this as a short-lived (dynamical age $\simeq 30 \mathrm{yr}$ ) spherical wind, powered by a high-mass object at its center (later detected in radio continuum observations by Curiel et al. 2002). Only five years after its discovery, this maser structure has already lost its spherical symmetry, probably due to its interaction 


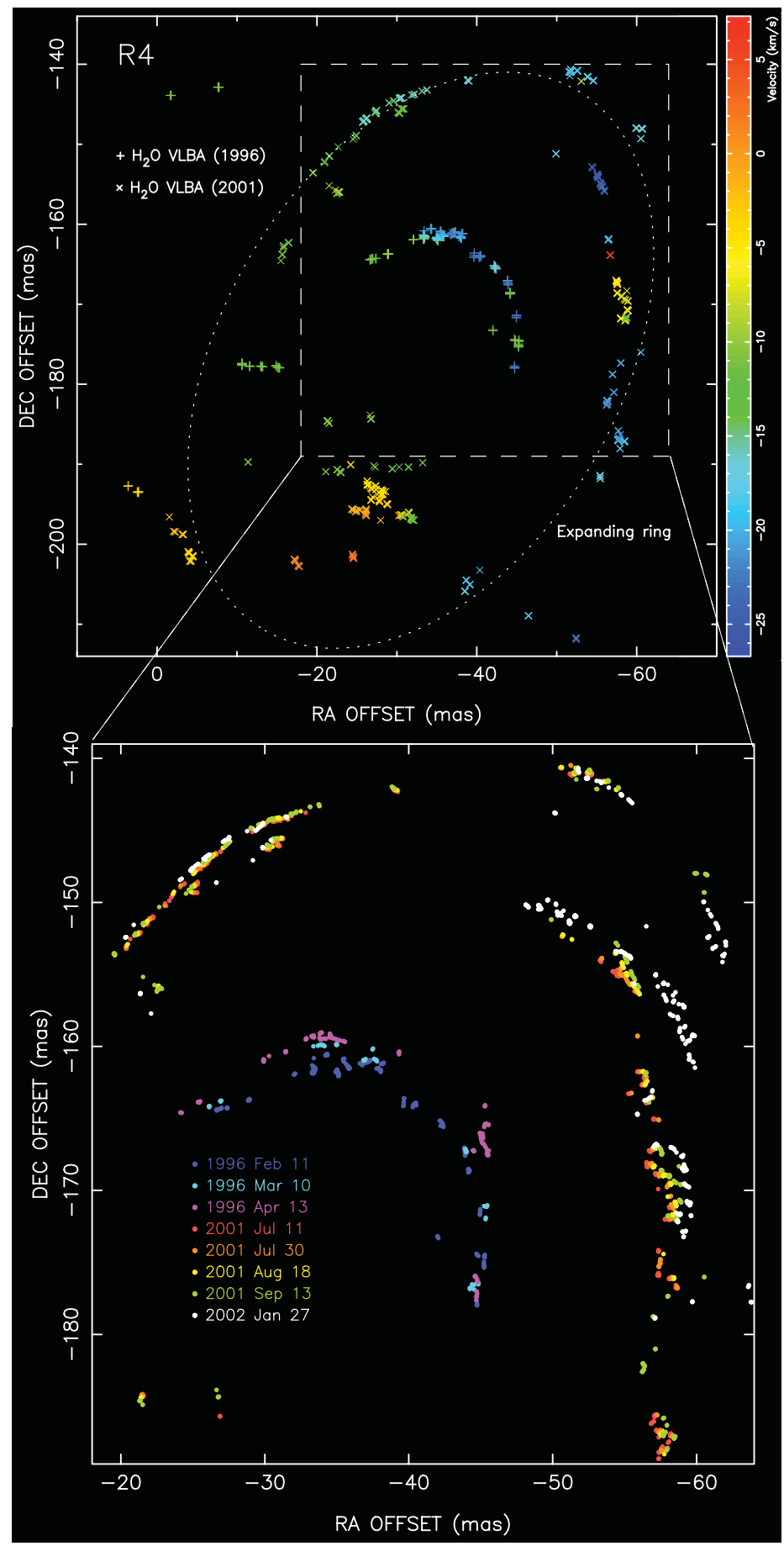

Figure 2. (Published online) Upper panel: Water maser positions measured in sub-region R4 of Cepheus A (see also Figure 1). Color code indicates the LSR radial velocity $(\mathrm{km} / \mathrm{s}$ ) of the masers. Lower panel: Zoom showing the evolution of the expanding motions in the sky for all the observed VLBA epochs. Color code indicates the epoch. (Figure from Torrelles et al. 2011). 


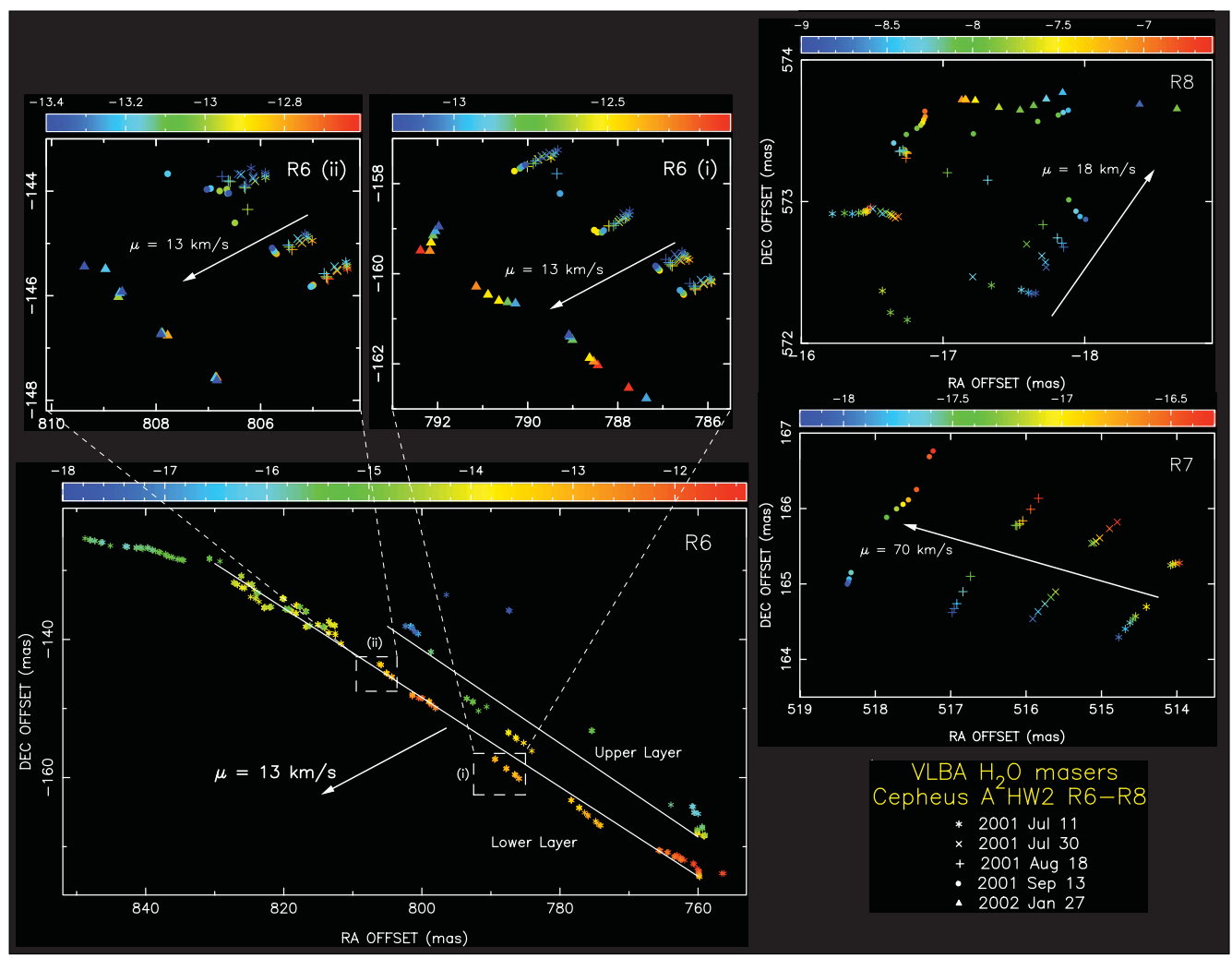

Figure 3. (Published online) Positions and proper motions of the $\mathrm{H}_{2}$ masers in sub-regions R6, R7, and R8 of Cepheus A (see also Figure 1). Color code indicates the LSR radial velocity $\left(\mathrm{km} \mathrm{s}^{-1}\right)$ of the masers. (Figure from Torrelles et al. 2011).

with the surrounding interstellar medium. The discovery of a nearly spherical ejection is especially relevant, since this phenomenon is difficult to explain in current models of star-formation, which assume that mass loss in YSOs is produced by the transformation of rotational energy in the disk into collimated outflows via magnetohydrodynamic mechanisms. A few other cases of nearly-isotropic ejections have been reported later in other star-forming regions (e.g., W75N, see §3). This indicates that uncollimated, episodic ejections may occur during the earliest stages of evolution of massive YSOs. The mechanism powering those ejections is still unclear.

While the maser structures in R4 and R5 are believed to be associated with different sources, the remaining ones (subregions R1 to R3, and R6 to R8) could trace an outflow powered by HW2. Significant differences in the magnitude and direction of the proper motions in these structures (Fig 3.), suggest that we can be witnessing the simultaneous presence of a collimated jet and a wide-angle outflow. In this scenario (Fig. 4), R6 (moving to the southeast at $\sim 13 \mathrm{~km} \mathrm{~s}^{-1}$ ) and $\mathrm{R} 8$ (to the northeast at $\sim 18 \mathrm{~km} \mathrm{~s}^{-1}$ ), would be tracing shock fronts at the walls of expanding cavities created by a wide-angle outflow from HW2, with an opening angle of $\simeq 100^{\circ}$. On the other hand, there is clearly a collimated jet traced by the radio continuum emission. The R7 masers, moving closer to the axis of this jet $\left(\simeq 30^{\circ}\right.$ from the axis), show expansion velocities of $\sim 70 \mathrm{~km} \mathrm{~s}^{-1}$, which are intermediate between those observed in $\mathrm{R} 6 / \mathrm{R} 8\left(\sim 13-18 \mathrm{~km} \mathrm{~s}^{-1}\right)$ and in the jet $\left(\sim 500 \mathrm{~km} \mathrm{~s}^{-1}\right)$. The R1-R3 masers, located on the opposite side from the central 


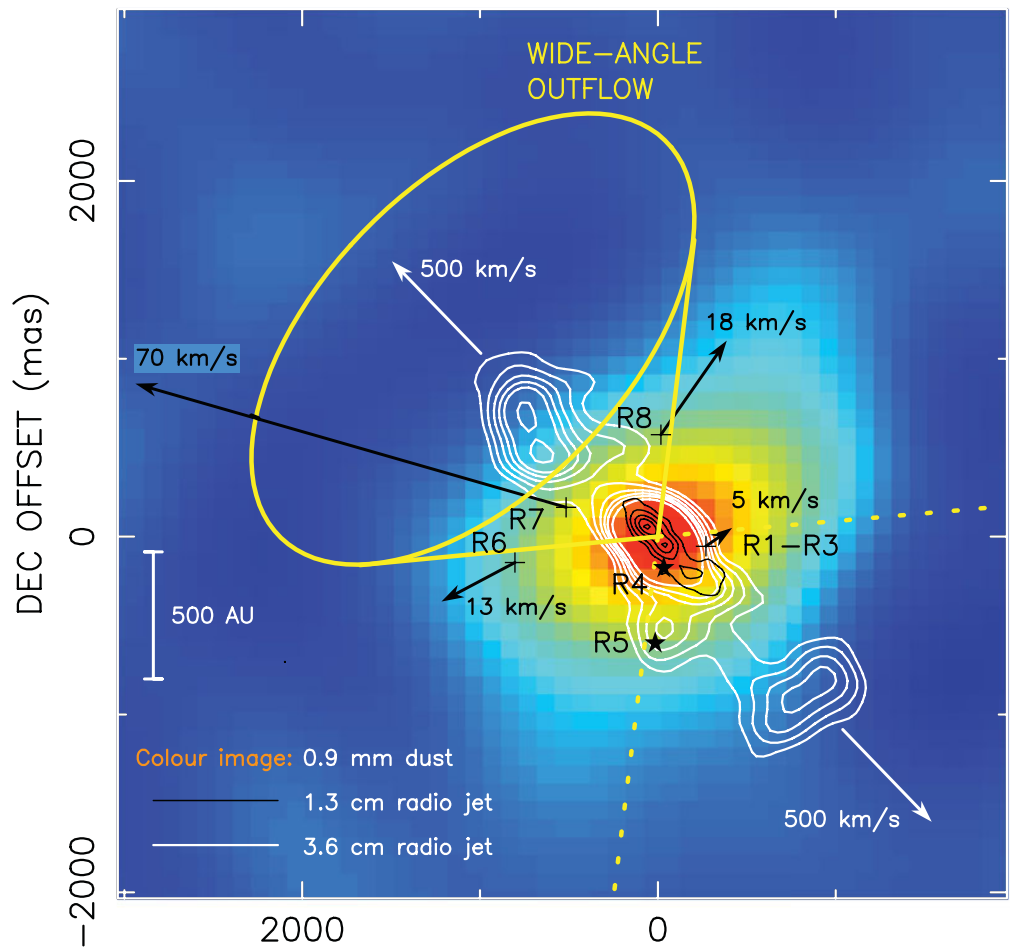

RA OFFSET (mas)

Figure 4. (Published online) Wide-angle outflow and jet in Cepheus A HW2. The radio jet (opening angle of $\sim 18^{\circ}$ ) exhibits ejections in opposite directions, moving away at $\sim 500 \mathrm{~km} \mathrm{~s}^{-1}$ from the central source, and is surrounded by a dust/molecular disk (Patel et al. 2005). R6, R8, and R1-3 trace emission fronts from the shocked walls of expanding cavities, created by the wide-angle wind of HW2 (opening angle of $\sim 100^{\circ}$ ). The R7 masers, with motions along an axis at an angle of $\sim 30^{\circ}$ with respect to the radio jet axis, are excited inside the cavity by the wide-angle wind. They exhibit higher velocity than R6, R8, and R1-3 (which are located at the expanding cavity walls) but lower than the velocity of the jet. The R6, R7, and R 8 masers (observed towards the blue-shifted lobe of the 1 arcmin, large-scale bipolar molecular outflow; Gómez et al. 1999) are blue-shifted with respect to the systemic velocity of the circumstellar disk, while R1-3 (observed towards the red-shifted lobe of the large-scale molecular outflow) are red-shifted. The position of the two massive YSOs required to excite the R4 and R5 maser structures are indicated by star symbols (see text). The star associated with R4 is not yet detected. (Figure and caption adapted from Torrelles et al. 2011, 2012).

source, moving at $\sim 5 \mathrm{~km} \mathrm{~s}^{-1}$ and with a difference in $\mathrm{PA}$ of $\simeq-80^{\circ}$ with respect to the radio jet direction, would represent the corresponding shocked walls of the southwestern cavities created by the wide-angle outflow.

The simultaneous presence of a collimated and a wide-angle outflow has been previously found in low-mass YSOs (e.g., see Velusamy et al. 2011 and references therein). Different theoretical models have been proposed to explain these two kind of outflows in low-mass YSOs (e.g., "X-wind", "Disk-wind" models; see Machida et al. 2008). The evidence that they are also found in a high-mass object, further reinforces that similar processes could be at work in both low- and high-mass star formation. Moreover, these observations in HW2 should provide important observational constraints for future models trying to reproduce the presence of outflows with different opening angles in high-mass YSOs. 


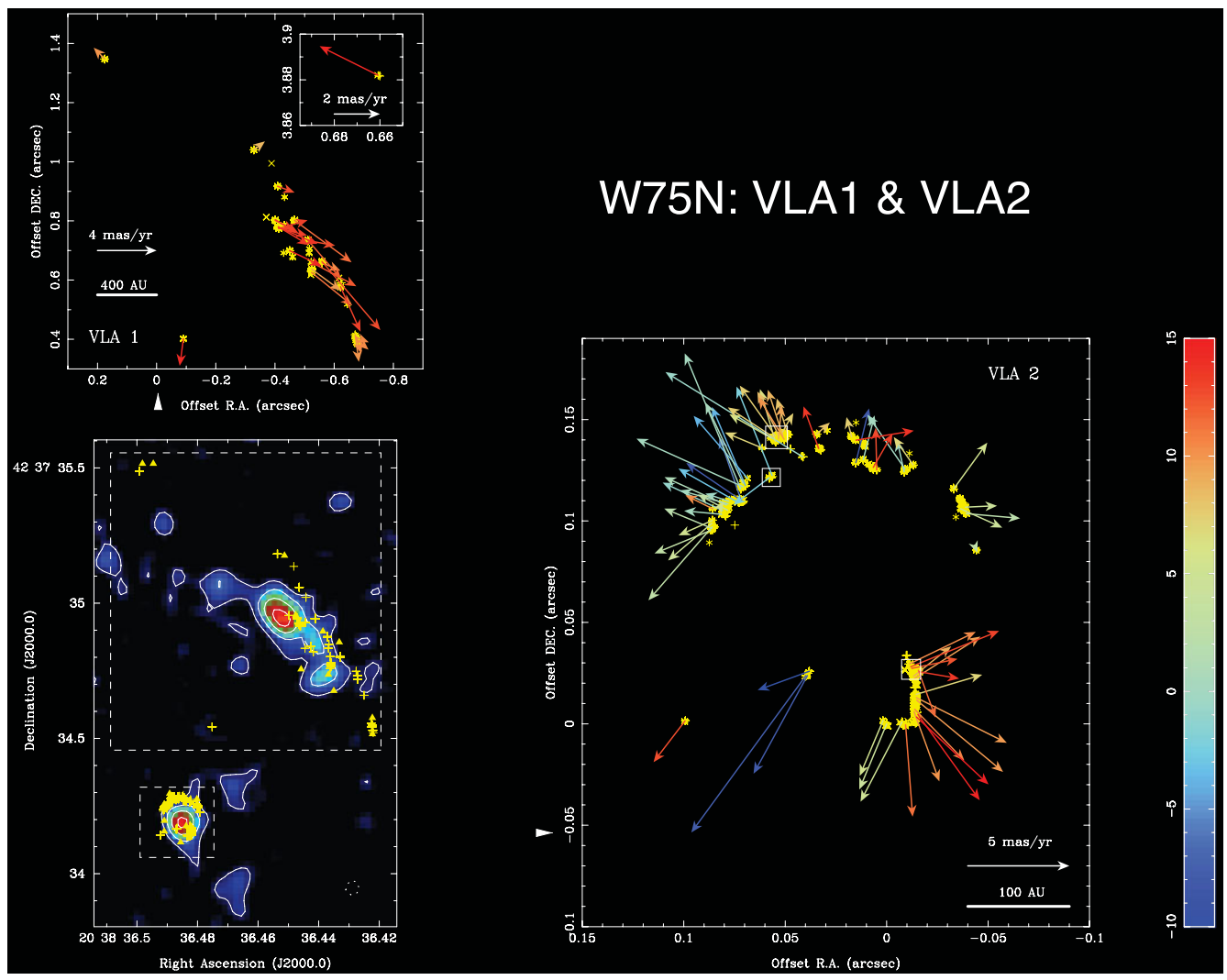

Figure 5. (Published online) Bottom left: $1.3 \mathrm{~cm}$ continuum contour map of VLA 1 and VLA 2. The positions of the $\mathrm{H}_{2} \mathrm{O}$ masers detected with the VLA (triangles) and VLBA (plus symbols) are indicated. Top left and bottom right: Proper motions (arrows) of the $\mathrm{H}_{2} \mathrm{O}$ masers in VLA 1 and VLA 2. Color code indicates the LSR velocity of the masers in $\mathrm{km} \mathrm{s}^{-1}$. (Figure from Torrelles et al. 2003, 2012).

\section{W75N}

We have focused our studies in three YSOs (VLA 1, VLA 2, VLA 3) in this region of high-mass star formation (see, e.g., Hunter et al. 1994, Torrelles et al. 1997, Persi et al. 2006, and Carrasco-González et al. 2010b). Despite being located within a region of only $1.5^{\prime \prime}$ (3000 AU at a distance of $2 \mathrm{kpc}$ ), they are believed to be in different evolutionary stages. In particular, VLA 1 and VLA 2 show distinctive mass-loss characteristics (see Figure 5): while in VLA1 the water masers observed with the VLBA, and the radio continuum emission trace a collimated jet of $\sim 2000$ AU size, the radio continuum emission in VLA 2 is compact, and the water masers seem to expand with no preferential direction, tracing a shell-like outflow of $\sim 160$ AU radius, moving at $\sim 30 \mathrm{~km} \mathrm{~s}^{-1}$ (dynamical age of $\simeq 13 \mathrm{yr}$ ). Given the close proximity between these sources (projected distance $1400 \mathrm{AU}$ ), they are likely to share a common molecular gas environment. This led Torrelles et al. (2003) to suggest that their different degree of outflow collimation is probably not due to ambient conditions, but to the different evolutionary stages of these sources, with VLA2 being younger. In this scenario, it is expected that the VLA 2 outflow would become more collimated as the source evolves. In fact, recent VLBA water maser observations (Surcis et al. 2011) indeed suggest that a jet is being formed in VLA 2. 
The case of VLA 2 is reminiscent of that of the R5 subregion in Cepheus A, suggesting the presence of non-collimated outflows in very early stages of high-mass YSOs. It is still unclear whether the observed non-collimated outflows are non-standard phenomena in particular types of sources, or if all massive YSOs undergo short-lived pulsed ejection phases with these type of processes. Nevertheless, their presence poses new challenges in our knowledge of the earliest stages of stellar evolution.

\section{Acknowledgement}

GA, RE, JFG, and JMT acknowledge support from MICINN (Spain) AYA2008-06189C03 and AYA2011-30228-C03 grants, co-funded with FEDER funds. SC acknowledges support from CONACyT (Mexico) 60581 and 168251 grants.

\section{References}

Anglada, G. 1996, ASPC, 93, 3

Carrasco-González, C., Rodríguez, L. F., Anglada, G., Martí, J., Torrelles, J. M., \& Osorio, M. 2010a, Science, 330, 1209

Carrasco-González, C. et al. 2011, RMxAC, 40, 229

Carrasco-González, C. et al. 2010b, AJ, 139, 2433

Chibueze, J. M. et al. 2012, ApJ, in press

Curiel, S. et al. 2006, ApJ, 638, 878

Curiel, S. et al. 2002, ApJ, 564, L35

Davies, B., Lumsden, S. L., Hoare, M. G., Oudmaijer, R. D., \& de Wit, W-J. 2010, MNRAS, 402, 1504

Elitzur, M., Hollenbach, D. J., \& McKee, C. F. 1992, ApJ, 394, 221

Fernández-López, M., Girart, J. M., Curiel, S., Gómez, Y., Ho, P. T. P., \& Patel, N. 2011, AJ, 142,97

Garay, G. \& Lizano, S. 1999, PASP, 111, 1049

Garay, G., Ramírez, S., Rodríguez, L. F., Curiel, S., \& Torrelles, J. M. 1996, 1996, ApJ, 459, 193

Goddi, C., Moscadelli, L., \& Sanna, A. 2011, A 6 A, 535, L8

Gómez, J. F. et al. 1999, ApJ, 514, 287

Hughes, V. A. \& Wouterloot, J. G. A. 1984, ApJ, 276, 204

Hunter, T. R., Taylor, G. B., Felli, M., \& Tofani, G. 1994, A\&A, 284, 215

Jiménez-Serra, I. et al. 2007, ApJ, 661, L187

Jiménez-Serra, I. et al. 2011, ApJ, 732, L27

Machida, M. N., Inutsuka, S.-i., \& Matsumoto, T. 2008, ApJ, 676, 1088

Moscadelli, L. et al. 2009, ApJ, 693, 406

Motogi, K. et al. 2008, MNRAS, 390, 523

Patel, N. A., Curiel, S., Sridharan, T. K., Zhang, Q., Hunter, T. R., Ho, P. T. P.., Torrelles, J. M., Moran, J. M., Gómez, J. F. G., \& Anglada, G. 2005, Nature, 437, 109

Persi, P., Tapia, M., \& Smith, H. A. 2006, A\&AA, 445, 971

Reid, M. J. \& Moran, J. M. 1981, ARAA, 19, 231

Rodríguez, L. F., Garay, G., Curiel, S., Ramírez, S., Torrelles, J. M., Gómez, Y., \& Velázquez, A. $1994, A p J, 430, \mathrm{~L} 65$

Sanna, A., Reid, M. J., Carrasco-González, C., Menten, K. M., Brunthaler, A., Moscadelli, L., \& Rygl, K. L. J. 2012, ApJ, 745, 191

Surcis, G.,Vlemmings, W. H. T., Curiel, S., Hutawarakorn Kramer, B., Torrelles, J. M., \& Sarma, A. P. 2011, A\& A, 527, 48

Torrelles, J. M., Gómez, J. F., Rodríguez, L. F., Ho, P. T. P., Curiel, S., \& Vázquez, R. 1997, ApJ, 489, 744

Torrelles, J. M., Patel, N., Anglada G., Gómez, J. F., Ho, P. T. P., Cantó, J., Curiel, S., Lara, L., Alberdi, A., Garay, G., \& Rodríguez, L. F. 2003, ApJ, 598, L115 
Torrelles, J. M., Patel, N. A., Curiel, S., Estalella, R., Gómez, J. F., Rodríguez, L. F., Cantó, J., Anglada, G., Vlemmings, W., Garay, G., Raga, A. C., \& Ho, P. T. P. 2011, MNRAS, 410,627

Torrelles, J. M., Patel, N. A., Curiel, S., Ho, P. T. P., Garay, G., \& Rodríguez, L. F. 2007, ApJ, 666, L37

Torrelles, J. M., Patel, N., Gómez, J. F., Ho, P. T. P., Rodríguez, L. F., Anglada, G., Garay, G., Greenhill, L., Curiel, S., \& Cantó, J. 2001a, Nature, 411, 277

Torrelles, J. M., Patel, N., Gómez, J. F., Ho, P. T. P., Rodríguez, L. F., Anglada, G., Garay, G., Greenhill, L., Curiel, S., \& Cantó, J. 2001b, ApJ, 560, 853

Torrelles, J. M., Patel, N., Curiel, S., Gómez, J. F., Anglada, G., \& Estalella, R. 2012, Boletín Asoc. Argentina de Astronomía, Eds. J.J. Clarià, A.E. Piatti, R. Barbá, P. Benaglia and F. Bareilles, No. 54, in press

Trinidad, M. et al. 2003, ApJ, 589, 386

Velusamy, T., Langer, W. D., Kumar, M. S. N., \& Grave, J. M. C. 2011, ApJ, 741, 60

Vlemmings, W. H. T. et al. 2010, MNRAS, 404, 134

Zapata, L. A. et al. 2009, ApJ, 698, 1422 